Research Paper

\title{
Evaluation of a novel monoclonal antibody against tumor-associated MUCl for diagnosis and prognosis of breast cancer
}

\author{
Natascha Stergiou ${ }^{\boxplus}$, Johannes Nagel ${ }^{2}$, Stefanie Pektor ${ }^{3}$, Anne-Sophie Heimes ${ }^{4}$, Jörg Jäkel ${ }^{5}$,Walburgis Brenner ${ }^{4}$, \\ Marcus Schmidt ${ }^{4}$, Matthias Miederer ${ }^{3}$, Horst Kunz ${ }^{6}$, Frank Roesch ${ }^{2 *}$, Edgar Schmitt ${ }^{\llbracket * *}$ \\ 1. Institute for Immunology, University Medical Center; \\ 2. Institute for Nuclear chemistry, Johannes-Gutenberg University; \\ 3. Clinic and Polyclinic for Nuclear Medicine, University Medical Center; \\ 4. Department of Obstetrics and Women's Health, University Medical Center, Johannes Gutenberg-University, Germany, \\ 5. Department of Pathology, University Medical Center; \\ 6. Institute for Organic Chemistry, Johannes-Gutenberg University. \\ *joint senior author \\ $\square$ Corresponding author: Prof. Dr. Edgar Schmitt, Langenbeckstraße 1, 55131 Mainz, Germany, phone: +49-6131-176195, fax: +49-6131-176202, \\ eschmitt@uni-mainz.de First author: Natascha Stergiou, n.stergiou@uni-mainz.de
}

(c) The author(s). This is an open access article distributed under the terms of the Creative Commons Attribution License (https://creativecommons.org/licenses/by/4.0/). See http://ivyspring.com/terms for full terms and conditions.

Received: 2019.04.24; Accepted: 2019.07.09; Published: 2019.08.14

\begin{abstract}
There is still a great unmet medical need concerning diagnosis and treatment of breast cancer which could be addressed by utilizing specific molecular targets. Tumor-associated $\mathrm{MUCl}$ is expressed on over $90 \%$ of all breast cancer entities and differs strongly from its physiological form on epithelial cells, therefore presenting a unique target for breast cancer diagnosis and antibody-mediated immune therapy. Utilizing an anti-tumor vaccine based on a synthetically prepared glycopeptide, we generated a monoclonal antibody (mAb) GGSK-1/30, selectively recognizing human tumor-associated $\mathrm{MUCl}$. This antibody targets exclusively tumor-associated $\mathrm{MUCl}$ in the absence of any binding to $\mathrm{MUCl}$ on healthy epithelial cells thus enabling the generation of breast tumor-specific radiolabeled immune therapeutic tools.

Methods: MAb GGSK-1/30 was used for immunohistochemical analysis of human breast cancer tissue. Its desferrioxamine (Df')-conjugate was synthesized and labelled with ${ }^{89} \mathrm{Zr}$. [ ${ }^{89} \mathrm{Zr}$ ]Zr-Df'-GGSK-1/30 was evaluated as a potential PET tracer. Binding and pharmacokinetic properties of [ $\left.{ }^{89} \mathrm{Zr}\right] \mathrm{Zr}$-Df'-GGSK-1/30 were analyzed in vitro using human and murine cell lines that express tumor-associated MUCl. Self-generated primary murine breast cancer cells expressing human tumor-associated $M U C l$ were transplanted subcutaneously in wild type and human MUCl-transgenic mice. The pharmacology of [ ${ }^{89} \mathrm{Zr}$ ]Zr-Df'-GGSK-1/30 was investigated using breast tumor-bearing mice in vivo by PET/MRT imaging as well as by ex vivo organ biodistribution analysis.

Results: The mAb GGSK-1/30 stained specifically human breast tumor tissue and can be possibly used to predict the severity of disease progression based on the expression of the tumor-associated $\mathrm{MUCl}$. For in vivo imaging, the $\mathrm{Df}$-conjugated $\mathrm{mAb}$ was radiolabeled with a radiochemical yield of $60 \%$, a radiochemical purity of $95 \%$ and an apparent specific activity of $6.1 \mathrm{GBq} / \mu \mathrm{mol}$. After $7 \mathrm{~d}$, stabilities of $84 \%$ in human serum and of $93 \%$ in saline were observed. In vitro cell studies showed strong binding to human tumor-associated $\mathrm{MUCl}$ expressing breast cancer cells. The breast tumor-bearing mice showed an in vivo tumor uptake of $>50 \% \mathrm{ID} / \mathrm{g}$ and clearly visible specific enrichment of the radioconjugate via PET/MRT.

Principal conclusions: Tumor-associated $\mathrm{MUCl}$ is a very important biomarker for breast cancer next to the traditional markers estrogen receptor (ER), progesterone receptor (PR) and HER/2-neu. The mAb GGSK-1/30 can be used for the diagnosis of over $90 \%$ of breast cancers, including triple negative breast cancer based on biopsy staining. Its radioimmunoconjugate represents a promising PET-tracer for breast cancer imaging selectively targeting breast cancer cells.
\end{abstract}

Key words: MUC1, breast cancer diagnosis, $\mathrm{mAb},{ }^{89} \mathrm{Zr}$ 


\section{Background}

Breast cancer is the most common cancer among women worldwide and the leading cause of cancer death among women (1). One in eight women suffers from breast cancer in her life (2). Breast cancer is usually detected either during a check-up before symptoms develop or after a woman has discovered a cancerous lump. If cancer is suspected, a microscopic analysis of the breast tissue is required for diagnosis, determination of breast cancer status and type of breast cancer. The tissue for microscopic analysis can be obtained by fine needle biopsy or surgery (American Cancer Society, Breast cancer risk factors). Traditional molecular markers for the characterization of breast cancer are estrogen receptor (ER), progesterone hormone receptor (PR) and Her2/neu; the standard method for their global assessment remains immunohistochemistry (3). The results of biopsy analysis are important for prognostic and therapeutic considerations (3). Due to the heterogeneity of breast cancer, these traditional markers are often not sufficient either for a precise prognosis or a sufficient statement about an adjuvant or neoadjuvant therapy. It is therefore essential to look for additional prognostic and predictive breast cancer markers that will be complementary in predicting clinical response to the available therapeutic modalities. In addition, there is a need to develop additional markers for such tumors that do not express ER, PR and or HER-2/neu (triple-negative breast cancer [TNBC]) (4). The aim is to bring additional markers in the clinic that predict the risk of recurrence and are helpful in decision making regarding appropriate treatment [8]. In 8 to $10 \%$ of women diagnosed with breast cancer, locoregional recurrences occur, and 15 to $30 \%$ develop distant metastases (1). A very promising marker to support breast cancer diagnosis and prognosis is the tumor-associated MUC1 ((TA)MUC1) (5-9). It is expressed in over $90 \%$ of all breast cancers (10) and even in $94 \%$ of TNBCs (11). Due to its characteristic aberrant glycosylation as a result of reduced activity of glycosyltransferases and accelerated activity of sialyltransferases in the MUC1 biosynthesis in breast cancer cells (12), breast-(TA)MUC1 represents a tumor-specific marker and target for therapy (13). Based on the aberrant glycan pattern, we synthesized human (TA)MUC1 (hu(TA)MUC1) glycopeptides derived from the tandem repeat (VNTR) region of this glycoprotein that correspond to the aberrant glycosylation pattern of (TA)MUC1. One specific hu(TA)MUC1 glycopeptide, 22mer huMUC1 peptide sequence of the VNTR region glycosylated with $\mathrm{ST}_{\mathrm{N}}$ on serine-17 located in the highly immune reactive GSTA motif, was conjugated to tetanus toxoid (TTox) forming an unique vaccine (5). Monoclonal antibodies (mAbs) were generated utilizing this vaccine. Among these, $m A b$ GGSK-1/30 was identified that specifically recognized the hu(TA)MUC1glycopeptide pattern on human breast cancer cells whereas fully glycosylated huMUC1 expressed by healthy breast epithelial cells was not recognized. We could also demonstrate that GGSK-1/30 showed stronger binding to these breast cancer cells than the commonly used and commercially available mAbs (SM3, HMFG1) (12). The aim of the current study was to evaluate the mAb GGSK-1/30 as a diagnostic and prognostic tool for breast cancer. Therefore, this $\mathrm{mAb}$ was evaluated by applying immune histochemical assays and molecular in vivo imaging using PET.

\section{Methods}

\section{Monoclonal antibody GGSK-1/30}

GGSK-1/30 was generated as described before after vaccination of $\mathrm{BALB} / \mathrm{c}$ mice with a $22 \mathrm{mer}$ huMUC1 peptide sequence of the VNTR region coupled to TTox $(5,12)$. GGSK-1/30 is of IgG1 isotype and was purified from hybridoma supernatant using Protein G and subsequently dialyzed versus PBS with the aid of a PD-10 desalting column (Sephadex G-25).

\section{Histological staining of human breast cancer specimens}

A panel of $144 \mathrm{HR}$ positive breast cancer tissue specimens of patients who were treated at the Department of Obstetrics and Gynecology of the Johannes Gutenberg University Mainz between the years 1987-2000 was examined for the expression of (TA)MUC1 by using GGSK-1/30 as a diagnostic tool. Patients' characteristics are given in Table $\mathbf{1}$. Immunohistochemical analyses were performed on 4 $\mu \mathrm{m}$ thick FFPE (Formalin-fixed paraffin embedded) sections according to standard procedures. In brief FFPE slides were subsequently deparaffinized using graded alcohol and xylene. Antigen retrieval reactions were performed in a steamer in citrate buffer of $\mathrm{pH} 10$ for 30 minutes. $3 \% \mathrm{H}_{2} \mathrm{O}_{2}$ solution was applied to block endogenous peroxidase at room temperature for 5 minutes. The samples were stained with GGSK-1/30 $(1 \mu \mathrm{g} / \mathrm{ml})$, followed by a polymeric biotin-free visualization system reaction (EnVision ${ }^{\mathrm{TM}}$, DAKO Diagnostic Company, Hamburg, Germany). In a next step, the sections were incubated with 3,3-diaminobenzidine (DAB; EnVision ${ }^{\mathrm{TM}}$, DAKO Diagnostic Company, Hamburg, Germany) for 5 minutes and counterstained with Mayer's haematoxylin solution. Paraffin sections of healthy breast tissue and paraffin sections of HR positive breast cancer tumours were examined. All slides were 
analyzed using a Leica light microscope (Leica Microsystem Vertrieb Company, Wetzlar, Germany) by two of the authors (ASH, JJ). Additionally, the magnitude of expression of (TA)MUC1 was scored in correlation of cumulative MFS and RFS according to the scoring system of Sinn et al. (14). This work was approved by the Landesärztekammer RheinlandPfalz, 837.287.05 (4945). All patients gave written informed consent before participating in this study. Follow-up data of all patients until 2014 were available and included the time period until development of metastases.

Table 1: Patientscharacteristics. Clinicopathological characteristics of hormone receptor positive patients who were treated at the Department of Obstetrics and Women's Health of University Medical Center Mainz $(\mathrm{N}=144)$. NST=invasive carcinoma of no special type, $\mathrm{PT}=$ primary tumor, $\mathrm{N}=$ number, (TA)MUCl=tumor-associated $\mathrm{MUCl}$.

\begin{tabular}{lll}
\hline Characteristics & $\mathrm{N}$ & $\%$ \\
\hline Tumor type & 95 & 66 \\
NST & 23 & 16 \\
Invasive lobular & 6 & 4.2 \\
Invasive tubular & 2 & 1.4 \\
mucinous & 18 & 12.4 \\
other & & \\
pT stage & 54 & 37.5 \\
pT1 & 74 & 51.4 \\
pT2 & 2 & 1.4 \\
pT3 & 14 & 9.7 \\
pT4 & & \\
Histological grade & 15 & 10.4 \\
G I & 105 & 72.9 \\
G II & 24 & 16.6 \\
G III & & \\
Estrogen receptor status & 0 & 0 \\
Negative & 144 & 100 \\
Positive & & \\
Progesterone receptor status & 17 & 11.9 \\
Negative & 127 & 88.1 \\
Positive & & \\
Lymph node status & 40 & 27.8 \\
Negative & 104 & 72.2 \\
Positive & & \\
(TA)MUC1 & & \\
(TA)MUC1 expression & 139 & 96.5 \\
no (TA)MUC1 expression & 5 & 3.5 \\
\hline
\end{tabular}

\section{Evaluation of immunostaining}

(TA)MUC1 expression was evaluated using an immunoreactivity score (IRS) as described by Sinn et al. (14) In brief, the percentage of positive tumor cells $(0 \%=0,1 \%-10 \%=1,11 \%-50 \%=2,51 \%-80 \%=3$, $81 \%-100 \%=4$ ) and the staining intensity (negative $=$ 0 , weak $=1$ moderate $=2$, strong $=3$ ) were multiplied, resulting in an immunoreactivity score (IRS) from 0 to 12. Cases with IRS 0-2 were considered as negative in terms of (TA)MUC1 expression due to possibly unspecific staining or material artifacts whereas cases with IRS 3-12 were considered as clearly visible and analyzable (TA)MUC1 expression. Kaplan-Meier Plots were performed to estimate survival rates. Significance levels were calculated using Log-Rank-Test. Statistical analysis was performed using the Graphpad Prism statistical software program, version 8.0.

\section{Animal breeding}

The transgenic C57BL/6-TG(MUC1)79.24 GEND/J (15) mice (huMUC1-tg, The Jackson laboratory) transgenically express the human MUC1 gene (huMUC1) and were housed and maintained in microisolator cages under specific pathogen-free conditions at the animal facility of Johannes Gutenberg-University following institutionally approved protocols (permission was obtained from the Landesuntersuchungsamt Koblenz, 23 177-07/G 08-1-019).

\section{Cell culture}

To obtain stable tumor cell lines from the autochthonous tumors of female PyMTxhuMUC1 mice and female PyMT mice (age of 18 weeks), tumor tissues were extracted, digested by collagenase A (Roche, 2mg/ml) and RQ1 DNAse (Promega, 1:2000) and cultured in IMDM (PAN Biotech, Aidenbach, Germany) + $5 \%$ FCS (Gibco ${ }^{\circledR}$, Life Technologies, Carlsbad, USA) $+1 \%$ glutamine (Roth, Karlsruhe, Deutschland) $+1 \%$ sodium pyruvate (Serva, Heidelberg, Deutschland). Stable PyMTxhuMUC1 tumor cells, expressing hu(TA)MUC1 and PyMT tumor cells that do not express hu(TA)MUC1 could be harvested after 6 weeks. In the first two weeks the cells were washed every third day to remove the tissue residues. After that primary tumour cells were cultured for additional 4 weeks to obtain the outgrowing adherent tumour cells and were passaged every time at a confluency of $70 \%$. Binding of GGSK-1/30 to both tumor cell lines (PyMTxhuMUC1 and PyMT) was analyzed via fluorescence-activated cell sorting (FACS) as follows: $2 \times 10^{5}$ tumor cells were incubated with $1 \mu \mathrm{g} / \mathrm{ml}$ GGSK-1/30 for $20 \mathrm{~min}$ at 4 ${ }^{\circ} \mathrm{C}$. The cells were washed two times with $100 \mu \mathrm{l}$ of PBS and incubated for 20 minutes at $4{ }^{\circ} \mathrm{C}$ with a secondary antibody goat-a-mouse-IgG Alexa Fluor 488 (dilution 1:1000 in PBS) in combination with a fixable viability dye eFluor780 (dilution 1:1000 in PBS) to exclude false positive dead cells. Tumor cells were washed twice with $100 \mu \mathrm{l}$ PBS followed by FACS analysis on a BD Biosciences FACSVerse machine.

\section{Conjugation of Df-Bz-NCS (Df) to GGSK-1/30 and radiolabeling with ${ }^{89} \mathbf{Z r}$}

All used chemicals were commercially available at Acros Organics, CheMatech, Fluka, SigmaAldrich or VWR and were used without further purification. 
GGSK-1/30 was coupled with Df' following a known procedure (16). In short, a ten-fold molar excess of $\mathrm{Df}^{\prime}$ (in $10 \mu \mathrm{l}$ DMSO) was added to the GGSK-1/30 (2 $\mathrm{mg} / \mathrm{ml}$ in $1 \mathrm{ml}$ PBS set to $\mathrm{pH} 9.0$ with $0.1 \mathrm{M} \mathrm{Na}_{2} \mathrm{CO}_{3}$ ) and incubated for $30 \mathrm{~min}$ at $37{ }^{\circ} \mathrm{C}$. The chelator-GGSK-1/30 conjugate was purified by size exclusion chromatography (SEC) using a PD-10 column and $0.25 \mathrm{M}$ sodium acetate buffer, $\mathrm{pH} 5.4$ as eluent.

For purification of conjugated and radiolabeled antibody, PD-10 desalting columns (GE Healthcare Life Science) were applied for dialysis versus $0.9 \%$ sodium chloride (Fresenius-Kabi) solution. For radiolabeling trace metal-free salts and water $(18 \mathrm{M} \Omega$ $\mathrm{cm}^{-1}$ ) were used. For radiolabeling, no-carrier-added (n.c.a.) ${ }^{89} \mathrm{Zr}$ (1 $\mathrm{M}$ oxalic acid) from PerkinElmer (Netherlands), trace metal-free salts and water (18 $\mathrm{M} \Omega$ $\mathrm{cm}^{-1}$ ) were used.

\section{Determination of chelator-to-mAb ratio (CAR)}

To determine the CAR, the conjugate was labeled according to aforementioned procedure (16) with a known nanomolar excess of zirconium oxalate solution (TraceCERT®, $1000 \mathrm{mg} / \mathrm{ml}$ ) spiked with ${ }^{89} \mathrm{Zr}$. Different molar ratios between $\mathrm{Zr}$ and GGSK-1/30 $\mathrm{mAb}$ were used to determine the number of chelators per antibody.

\section{Preparation of [ ${ }^{89 Z} \mathrm{Zr}$ ]Zr-Df-GGSK-1/30 and analytical quality control of [ ${ }^{89} \mathrm{Zr}$ ]Zr-Df-GGSK-1/30}

Df'-GGSK-1/30 was labeled according to aforementioned method (17). In short, Df'-GGSK-1/30 was radiolabeled with ${ }^{89} \mathrm{Zr}$ in HEPES buffer $(0.5 \mathrm{M}$, $\mathrm{pH} 7$ ) at room temperature in a volume of $2.5-3 \mathrm{ml}$ under gentle stirring for $90 \mathrm{~min}$. Radiochemical yield (RCY) was determined by radio thin layer chromatography (using Merck Silica F254 TLC plates with citrate buffer, $(0.01 \mathrm{M}, \mathrm{pH} 4)$ analyzed with the radio detector GABI STAR (Raytest, SI Figure 1). The radiolabeled compound was purified by PD-10 column using a $0.9 \%$ sodium chloride solution as eluent. HPLC monitoring was performed on a HPLC system from Merck (LaChrom; pump: Hitachi L7100; UV-detector: L7400) using a BioSep SEC-S 2000 column (Phenomenex ${ }^{\circledR}$ ) with $0.05 \mathrm{M}$ sodium phosphate $(\mathrm{pH} 7)$ as mobile phase $(1 \mathrm{ml} / \mathrm{min})$ (SI Figure 2).

\section{In vitro stability test of [ $\left.{ }^{89} \mathrm{Zr}\right] \mathrm{Zr}$-Df-GGSK- $1 / 30$}

In vitro stability studies of $\left[{ }^{89} \mathrm{Zr}\right] \mathrm{Zr}-\mathrm{Df} \mathbf{f}^{\prime}-\mathrm{GGSK}-$ $1 / 30$ were performed in human serum (Sigma-Aldrich ${ }^{\circledR}$, from human male AB plasma) and sodium chloride $(0.9 \%)(\mathrm{n}=3)$. The samples were incubated at $37^{\circ} \mathrm{C}$ and aliquots of $2 \mu 1$ were analyzed at various time points $(1 \mathrm{~d}, 3 \mathrm{~d}, 7 \mathrm{~d})$ via radio-TLC using citrate buffer (SI Figure 3).

\section{In vitro binding studies of [ $\left.{ }^{89} \mathrm{Zr}\right] \mathrm{Zr}$-Df-GGSK- $1 / 30$}

For in vitro binding studies different concentration of $\quad\left[{ }^{89} \mathrm{Zr}\right] \mathrm{Zr}-\mathrm{Df}{ }^{\prime}-\mathrm{GGSK}-1 / 30 \quad(0.125-$ $1 \mu \mathrm{g} / \mathrm{ml}$ ) were incubated with $2 \times 10^{5}$ tumor cells for 30 $\min$ at $37^{\circ} \mathrm{C}$. The supernatant was removed, the cell surface washed twice with PBS buffer. The washing solution was kept to detect the unbound antibody. Hence, the radioactivity of the cells and the washing solution was detected with a gamma counter (PerkinElmer Wizard2). The ratio cells/washing solution $\times 100$ resulted in binding/\%.

\section{Inoculation of tumor cells}

For all in vivo kinetic experiments 10 weeks old female C57BL/6N mice (Janvier) were used. Either $1 \times 10^{6}$ PyMTxhuMUC1 tumor cells or $2 \times 10^{5}$ PyMT tumor cells were subcutaneously (s.c.) inoculated in the right flank. To determine the biodistribution of the $\mathrm{mAb}$ in mice expressing huMUC1 on every epithelial cell, mimicking the human background, $1 \times 10^{6}$ PyMTxhuMUC1 tumor cells were inoculated in nine 10 weeks old huMUC1-transgenic mice. The tumor growth was observed every 3 days.

\section{Animal studies}

$21 \mathrm{~d}$ after inoculation (tumor size $40 \mathrm{~mm}^{2}$ on average), $\quad 50-80 \mu \mathrm{g} \quad\left(0.5-2.5 \mathrm{MBq}{ }^{89} \mathrm{Zr}\right)$ of the radioconjugates were administered intraperitoneal (i.p.) in 250-300 $\mu 1$ PBS. Mice were anesthetized with isoflurane (2 vol\%)/ oxygen gas mixture).

\section{Ex vivo biodistribution}

All mice were sacrificed and dissected after $24 \mathrm{~h}$, $48 \mathrm{~h}, 72 \mathrm{~h}$ and $10 \mathrm{~d}$. Blood, tumor, normal tissue and gastrointestinal contents were weighted and the amount of radioactivity in each tissue was measured in a gamma-counter (PerkinElmer Wizard2). Radioactivity uptake was calculated as the percentage of the injected dose per gram of tissue (\% ID/g(tissue)).

\section{In vivo small animal PET studies}

Small animal PET studies were carried out $72 \mathrm{~h}$ after application of radioconjugates regarding the highest enrichment of the mAbs in the tumors at this time point. All scans were performed in head-first-prone position in a PET-MRI scanner (Mediso NanoScan, Mediso, Hungary). In some experiments MRI measurements (Material Map) were first performed for co-registration of the PET scan (3D 
Gradient Echo External Averaging (GRE-EXT), Multi Field of View (FOV); slice thickness: 0,6 mm; TE: 2 ms; TR: 15 ms; flip angle: $25 \mathrm{deg}$ ) followed by a static PET scan (collecting 20 million events). PET data were reconstructed with Teratomo 3D (4 iterations, 6 subsets, voxel size $0.4 \mathrm{~mm}$ ), co-registered to the MR and analyzed with PMOD software (version 3.6, PMOD Technologies LLC).

\section{Results}

In a recent publication, we have already shown that our mAb GGSK-1/30 stained highly specific tumorous tissue from TNBC patients (18). In this study, we examined a very large group of human hormone receptor positive (HR positive) breast cancer biopsies with the mAb GGSK-1/30 in cooperation with the Department of Obstetrics and Women's Health of the University Medical Center in Mainz. HR positive breast cancer patients represent the largest group of patients with $75 \%$ (19). The immunohistochemistry (IHC) analyses demonstrated again the diagnostic use of mAb GGSK-1/30 for the detection of breast cancer tissue. Therefore, 10 sections of healthy human breast tissue (Figure 1A) and 144 sections of HR positive breast cancer tissue (Figure 1B) were stained with GGSK-1/30. The staining of healthy breast tissue with GGSK-1/30 was negative in all cases. By contrast $96.5 \%$ of all breast cancer tissue sections were clearly positively stained with GGSK-1/30, 3.5\% were negative.
A

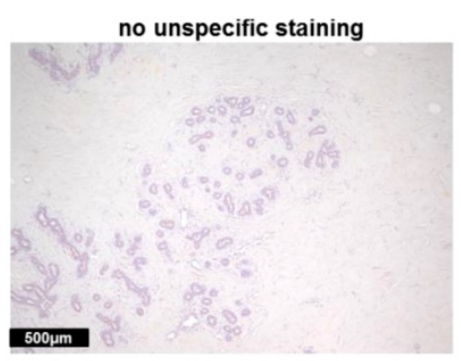

B
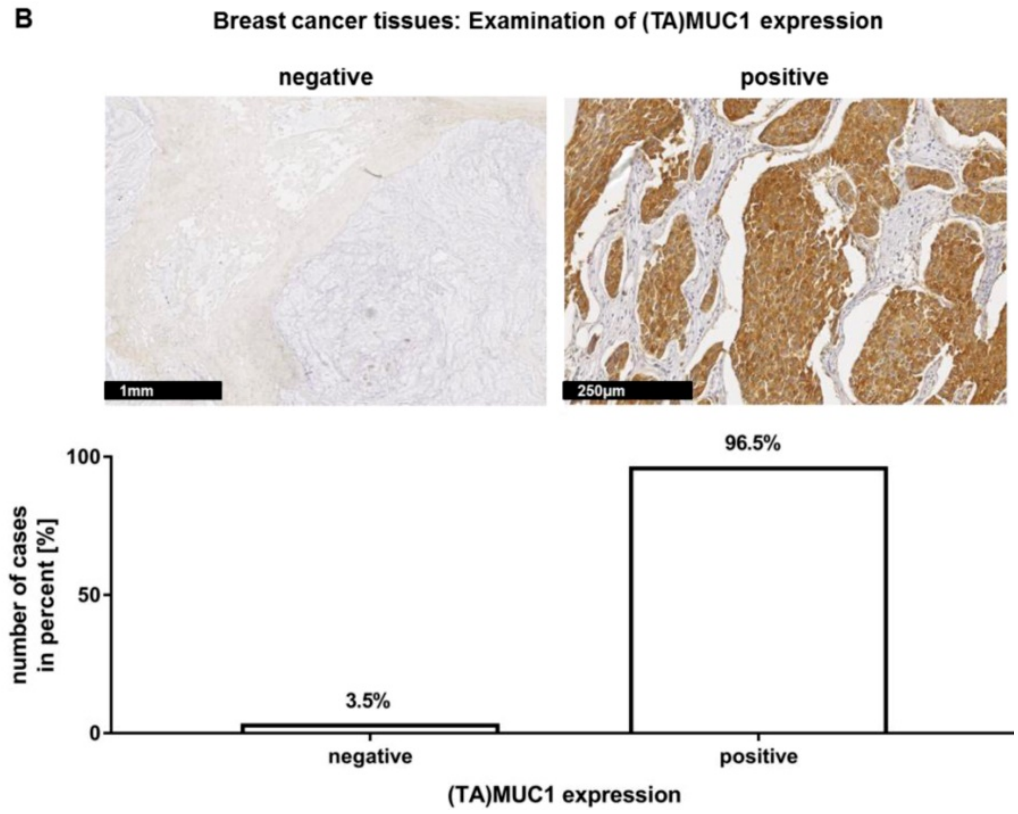

Figure 1: Immunohistochemical staining of (TA)MUC1 with GGSK-1/30 in human breast cancer specimens. A collective of breast cancer tissue sections from 144 patients was examined for (TA)MUCl specific staining. Paraffin sections of healthy breast tissue (A) and paraffin sections of hormone receptor positive breast tumors (B) were examined. Representative examples from 144 breast cancer tissue sections and 10 healthy mammary tissue sections are shown. (TA)MUCl=Tumor-associated MUCl.
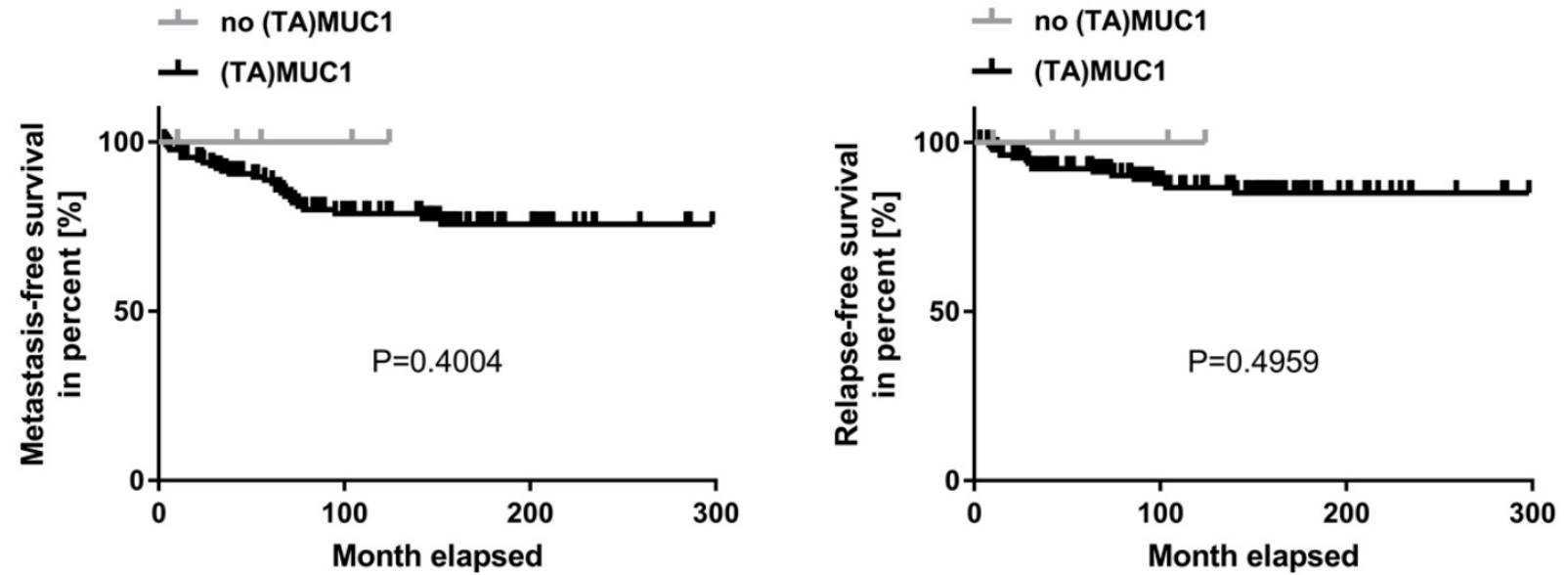

Figure 2: Kaplan-Meier curve of metastasis-free and relapse-free survival comparing (TA)MUCl expression and no (TA)MUC1 expression. A collective of breast cancer tissue sections from 144 patients was examined for (TA)MUCl specific staining. The status of (TA)MUC1 expression was correlated to metastasis-free or relapse-free survival with follow up patient data. Significance levels were calculated using Log-Rank-Test. (TA)MUC1=Tumor-associated MUC1. 
A

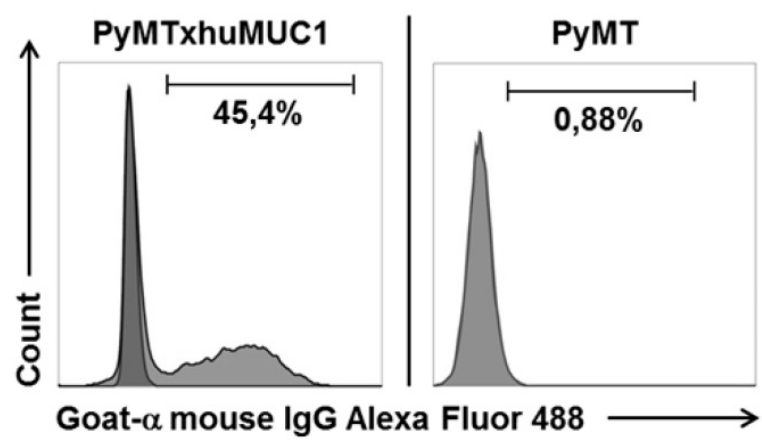

B

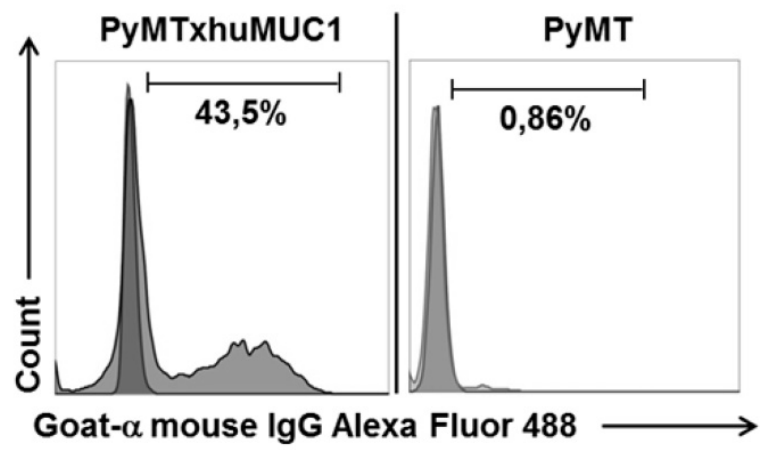

Figure 3: Specific binding of mAb GGSK-1/30 and to huMUC1-expressing tumor cells. Murine huMUC1-expressing PyMTxhuMUC1 tumor cells and PyMT tumor

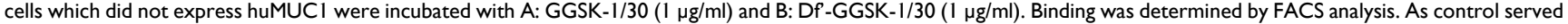
the unspecific binding of the secondary antibody goat a-mouse IgG Alexa Fluor 488 to the tumor cells (dark grey).

A correlation analysis was carried out concerning (TA)MUC1 expression in relation to metastasis-free survival (MFS) and relapse-free survival (RFS) as well. The patient's cumulative MFS and RFS suggest that the (TA)MUC1 expression may be correlated with a comparatively poor prognosis (Figure 2). However, our data failed to show any prognostic significance of (TA)MUC1 expression neither in terms of MFS nor in terms of RFS in this cohort of HR positive breast cancer samples. This might be due to the small sample number in the subgroup of (TA)MUC1 negative expression. The immune histochemical data which confirmed an exclusive binding of mAb GGSK-1/30 to (TA)MUC1-glycopeptides indicated that this $\mathrm{mAb}$ can be used for the diagnosis of breast cancer. Whether (TA)MUC1 expression could be used as a prognostic marker, should be evaluated on the basis of a considerable larger collective.

After breast cancer diagnosis, positron emission tomography (PET) can be used to determine whether the cancer has spread to the lymph nodes or to other organs. During therapy, PET imaging can also be used in monitoring the effectiveness and response to the treatment(s). Women who have completed treatment, but remain at high risk for recurrence might also be good candidates for follow-up PET screening. The best-studied and most widely used clinical-grade PET tracer is 2-Fluor-2-desoxy-D-glucose (FDG) (20). However, false negative PET results due to low FDG uptake can easily occur in certain types of breast cancer, such as invasive lobular carcinoma. In addition, false positive results can occur during an inflammation. Therefore, the development of additional PET biomarkers is needed, which also aims to improve patient restaging information and to evaluate therapeutic efficacy (21). Thus, we analyzed whether the GGSK-1/30 mAb was applicable as a biomolecular imaging agent selectively binding to
(TA)MUC1 in a preclinical breast cancer mouse model. We established a transplantable breast tumor model with murine breast cancer cells that express human MUC1 (PyMTxhuMUC1 cells) (18). These primary cancer cell lines were established from tumor biopsies of F1 PyMT (Tg(MMTVPyMT)634Mul (22)) crossbred with human MUC1 (C57BL/6-TG(MUC1) 79.24GEND/J (15)) double transgenic mice (PyMTxhuMUC1 mice). Using FACS analysis we could show that the mAb GGSK-1/30 specifically binds to these human MUC1 expressing murine cancer cells (Figure 3A). As negative control served murine breast cancer cells that did not express human MUC1 after isolation from PyMT (Tg(MMTVPyMT)634Mul mice. For in vivo imaging studies GGSK-1/30 was radiolabeled with ${ }^{89} \mathrm{Zr}$. Long-lived PET nuclides like ${ }^{89} \mathrm{Zr}$ are of great interest for ImmunoPET imaging and are ideal candidates for radiolabeling mAbs $(23,24)$. ${ }^{89} \mathrm{Zr}$ is advantageous because it remains in the cells after internalization of the $\mathrm{mAb}$ conjugate, resulting in improved tumor image contrast accumulation. In addition, its half-life of about 78 hours allows binding to the target over a longer period of time, which correlates well with the long biological half-life of mAbs (25). We used hydroxamate groups of desferrioxamine (Df') as chelating agent for ${ }^{89} \mathrm{Zr}$. Coupling of the Df' chelator resulted in a ratio of 4.2 chelator moieties per antibody. Binding of Df'-GGSK-1/30 mAb to PyMTxhuMUC1 tumor cell lines was analyzed by FACS analysis. Figure 3B demonstrates that binding of Df'-GGSK-1/30 to PyMTxhuMUC1 tumor cells was not impaired upon coupling of the Df'.

The radiolabeling of Df'-GGSK-1/30 with ${ }^{89} \mathrm{Zr}$ was performed at room temperature (26) with an overall yield of $73 \%$ (SI Figure 1). After purification with a PD-10 desalting column, the radiochemical purity of [ $\left.{ }^{89} \mathrm{Zr}\right] \mathrm{Zr}-\mathrm{Df} \mathrm{f}^{\prime}-\mathrm{GGSK}-1 / 30$ exceeded $95 \%$ with an apparent specific activity of $6.1 \mathrm{GBq} / \mu \mathrm{mol}$ (SI 
Figure 2). [ $\left.{ }^{89} \mathrm{Zr}\right] \mathrm{Zr}-\mathrm{Df}$ '-GGSK-1/30 exhibited a high stability of $>90 \%$ after 3 days in human serum (HS) and $0.9 \% \mathrm{NaCl}$ solution (SI Figure 3). In $0.9 \% \mathrm{NaCl}$ solution [ $\left.{ }^{89} \mathrm{Zr}\right] \mathrm{Zr}-\mathrm{DF}^{\prime}$-GGSK-1/30 remained stable even after 3 days, while a slight decrease to $83 \%$ intact conjugate after 7 days was observed in HS. In vitro binding of [ $\left.{ }^{89} \mathrm{Zr}\right] \mathrm{Zr}$-Df'-GGSK-1/30 to hu(TA)MUC1 was evaluated to verify the diagnostic potency of the radiolabeled conjugate in respect to first in vivo studies. A dose-dependent (>15\%) binding to murine PyMTxhuMUC1 breast tumor cells which express hu(TA)MUC1 could be observed, while [89 Zr]Zr-Df'-GGSK-1/30 did not bind to the murine PyMT breast tumor cells (Figure 4).

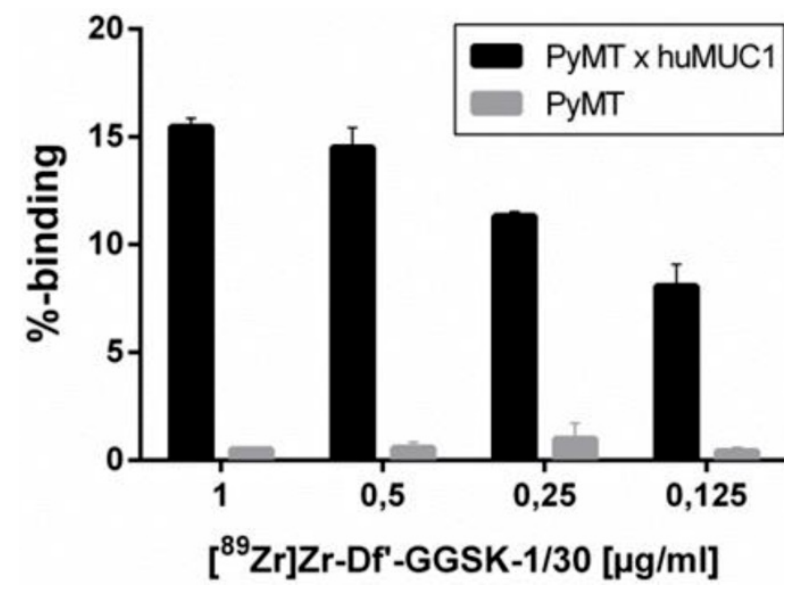

Figure 4: Specific binding of [ $\left.{ }^{89} \mathrm{Zr}\right] \mathrm{Zr}$-Df-GGSK-1/30 mAb to huMUC1-expressing tumor cells. Murine PyMTxhuMUC1 tumor cells were incubated in the presence of decreasing concentrations of [ $\left.{ }^{89} \mathrm{Zr}\right] \mathrm{Zr}$-Df-GGSK-1/30 $(1-0.125 \mu \mathrm{g} / \mathrm{ml})$ and binding was determined by FACS analysis. Murine PyMT tumor cell line which does not express huMUCl served as negative controls.

To confirm the specificity of [ $\left.{ }^{89} \mathrm{Zr}\right] \mathrm{Zr}-\mathrm{DF}^{\prime}-$ GGSK-1/30 for hu(TA)MUC1 in vivo and to assess its usage as a future diagnostic tool for huMUC1-expressing breast cancers, the radioconjugate was administered i.p. in C57BL/6N mice bearing PyMTxhuMUC1 breast tumor cells subcutaneously on the right flank. After 24 hours, 48 hours, 72 hours and 10 days analyses of the biodistribution were carried out. Additionally, PET imaging was performed after 72 hours (Figure 5). The highest amount of $\left[{ }^{89} \mathrm{Zr}\right] \mathrm{Zr}-\mathrm{Df} \mathbf{f}^{\prime}$-GGSK-1/30 was detected after 72 hours in the tumor $(>55 \% \mathrm{ID} / \mathrm{g})$. Uptake values in other tissues (lung, heart, spleen, pancreas, stomach, intestines, kidneys, lymph nodes, mammary glands, muscle) were below 20 $\%$ ID/g(tissue) (SI Figure 4). The concentration of the [ ${ }^{89} \mathrm{Zr}$ ]Zr-Df'-GGSK-1/30 in blood decreased steadily while increasing amounts of [ $\left.{ }^{89} \mathrm{Zr}\right] \mathrm{Zr}-\mathrm{Df}{ }^{\prime}-\mathrm{GGSK}-1 / 30$ accumulated in the tumor. The radioconjugate showed predominant hepatobiliary excretion with increasing uptake values over time from 22 to $38 \%$ ID/g (liver). The uptake values in bone tissues steadily increased (Figure 5A) due to the slight degradation of the Zr-Df'-complex in vivo, which is known for ${ }^{89} \mathrm{Zr}$-radiolabeled $\mathrm{Df}^{\prime}$-conjugated antibodies (26-28). An exact calculation of the tumor-to-tissue ratio revealed a comparatively high tumor accumulation (Figure 5B). In agreement with these findings, PET imaging after 72 hours demonstrated a strong accumulation of [ $\left.{ }^{89} \mathrm{Zr}\right] \mathrm{Zr}-\mathrm{Df}$-GGSK-1/30 in the tumor (Figure 5C).

Potential diagnostic and therapeutic usage of [ $\left.{ }^{89} \mathrm{Zr}\right] \mathrm{Zr}$-Df'-GGSK-1/30 in breast cancer patients requires that unspecific binding to normally glycosylated huMUC1 on healthy tissue should be largely excluded. To investigate impact of unspecific binding on tumor accumulation, PyMTxhuMUC1 tumor cells were transplanted into huMUC1transgenic mice carrying huMUC1 on all epithelial cells. Maximum accumulation of [ $\left.{ }^{89} \mathrm{Zr}\right] \mathrm{Zr}-\mathrm{Df} \mathrm{f}^{\prime}$-GGSK$1 / 30$ in the tumor could be observed 72 hours after i.p. application in wild type mice. Therefore, analyses of in vivo biodistribution and PET imaging were performed in the huMUC1-transgenic mice at this time point. As additional control concerning the specificity of the mAb GGSK-1/30 for hu(TA)MUC1, mice were injected with [ $\left.{ }^{89} \mathrm{Zr}\right] \mathrm{Zr}$-Df'-GGSK-1/30 which had been blocked before with a 1200 -fold molar excess of its specific hu(TA)MUC1-glycopeptide antigen (12). Figure 6A shows the uptake values of [ $\left.{ }^{89} \mathrm{Zr}\right] \mathrm{Zr}-\mathrm{Df}$ '-GGSK-1/30 (\%ID/g (tissue)) for blood, spleen, liver, bones, tumor tissue and mammary glands. The low unspecific uptake values (less than 10 $\% \mathrm{ID} / \mathrm{g}$ ) of the radiolabeled $\mathrm{mAb}$ in the mammary glands, which overexpress normal huMUC1 are similar to other non-target tissues, whereas up to 65 $\% \mathrm{ID} / \mathrm{g}$ could be observed in the tumor. An exact calculation of the tumor-to-tissue ratio revealed a comparatively high tumor accumulation (Figure 6B). These data demonstrate again that the unique $\mathrm{mAb}$ GGSK-1/30 binds to hu(TA)MUC1 containing the aberrant glycosylation pattern whereas normal huMUC1 expressed on healthy cells are not bound. Blocking of radiolabeled $\mathrm{mAb}$ by its specific hu(TA)MUC1-glycopeptide prevented binding to tumor tissue demonstrating again the antigen specificity of GGSK-1/30 for hu(TA)MUC1. A detailed presentation of the biodistribution of the [ $\left.{ }^{89} \mathrm{Zr}\right] \mathrm{Zr}-\mathrm{Df} \mathrm{f}^{\prime}-\mathrm{GGSK}-1 / 30$ is shown in SI Figure 5. These analyses revealed an exceptional specificity of mAb GGSK-1/30 for hu(TA)MUC1 in vivo and were further supported in vivo by PET imaging (Figure 6C). 
A

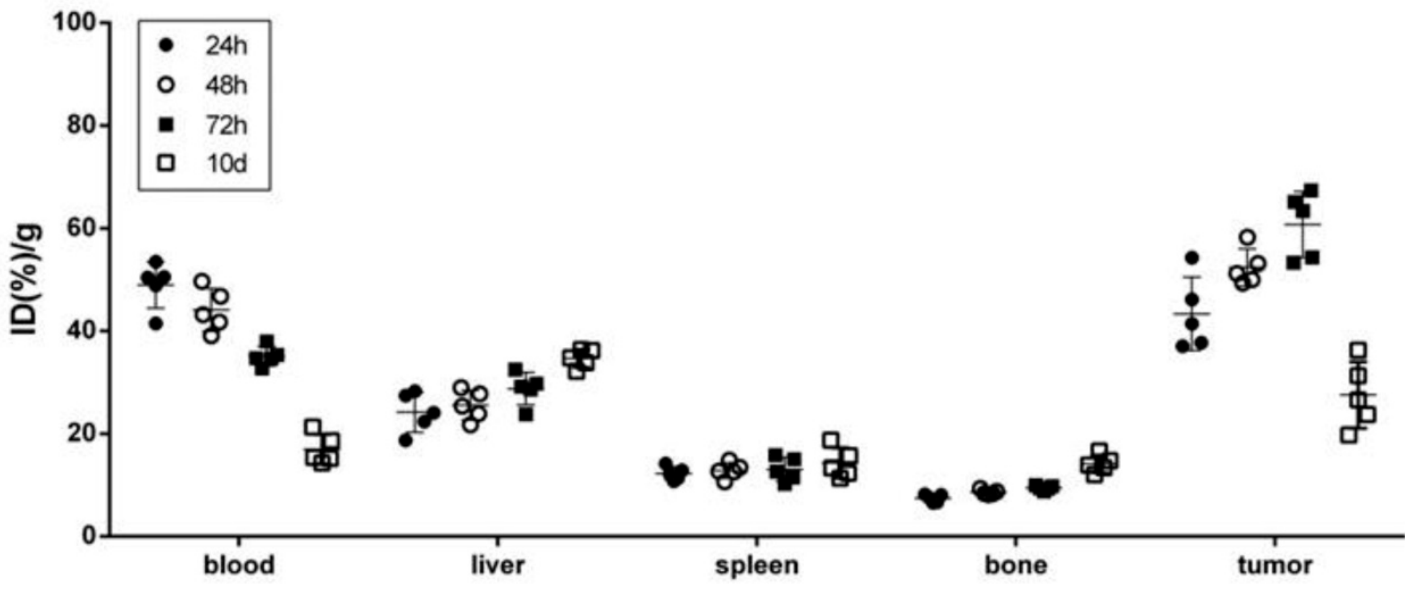

B

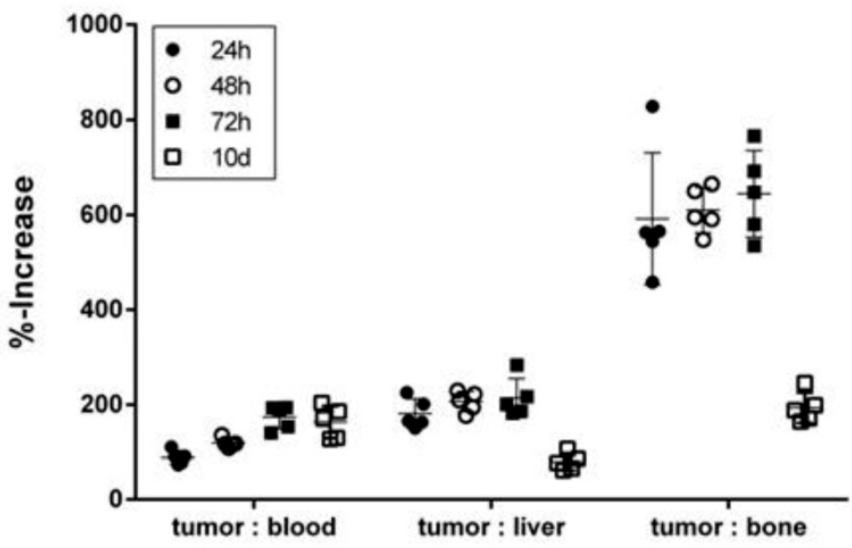

C

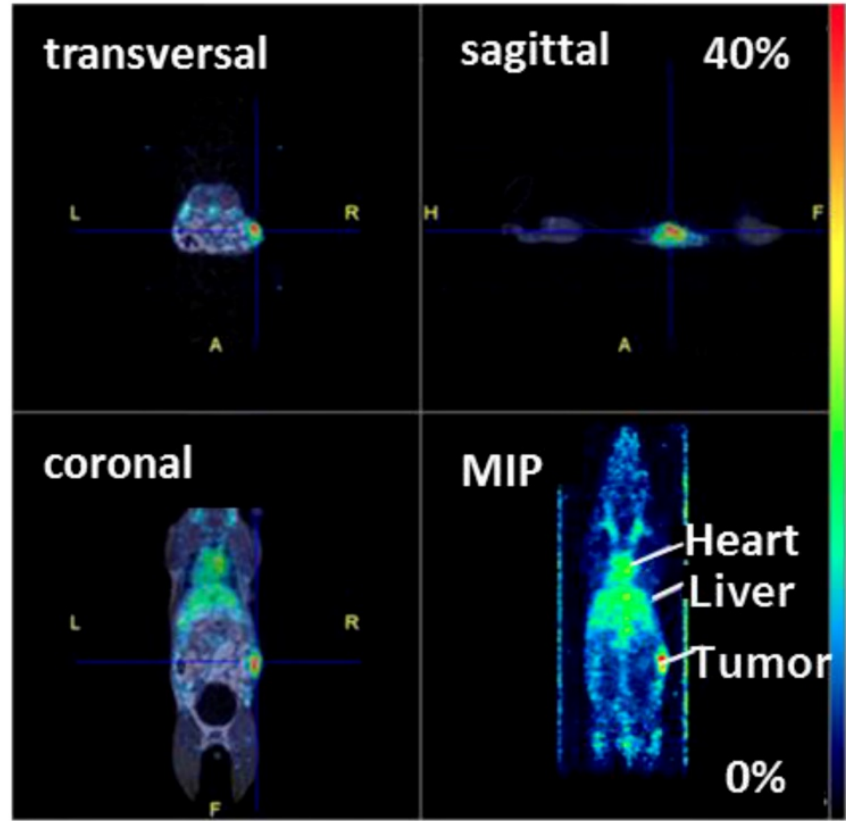

Figure 5: Biodistribution of [89Zr]Zr-Df-GGSK-1/30 in wild type mice bearing PyMTxhuMUC1 breast tumors. C57BL/6N mice bearing a PyMTxhuMUC1 breast tumor transplant subcutaneously on the right flank were treated with $\left[{ }^{89} \mathrm{Zr}\right] \mathrm{Zr}$-Df-GGSK-1/30 mAb $(80 \mu \mathrm{g}, 1 \mathrm{MBq})$ i.p. ( $\left.\mathrm{n}=20\right)$. After $24 \mathrm{~h}, 48 \mathrm{~h}, 72 \mathrm{~h}$ and $10 \mathrm{~d}$ the distribution of the radioconjugate $(\mathrm{A})$ and the tumor/non-target-tissue ratios $(\mathrm{B})$ were determined $(\mathrm{ID}(\%) / \mathrm{g}(\mathrm{tumor}): \mathrm{ID}(\%) / \mathrm{g}(\mathrm{blood}$, liver, bone)*100=\%-increase). (C) PET images from a representative breast tumor-bearing mouse after 72 h. Abbreviations: tu.: tumor, he: heart, li.: liver; MIP: Maximum Intensity Projection. 
A

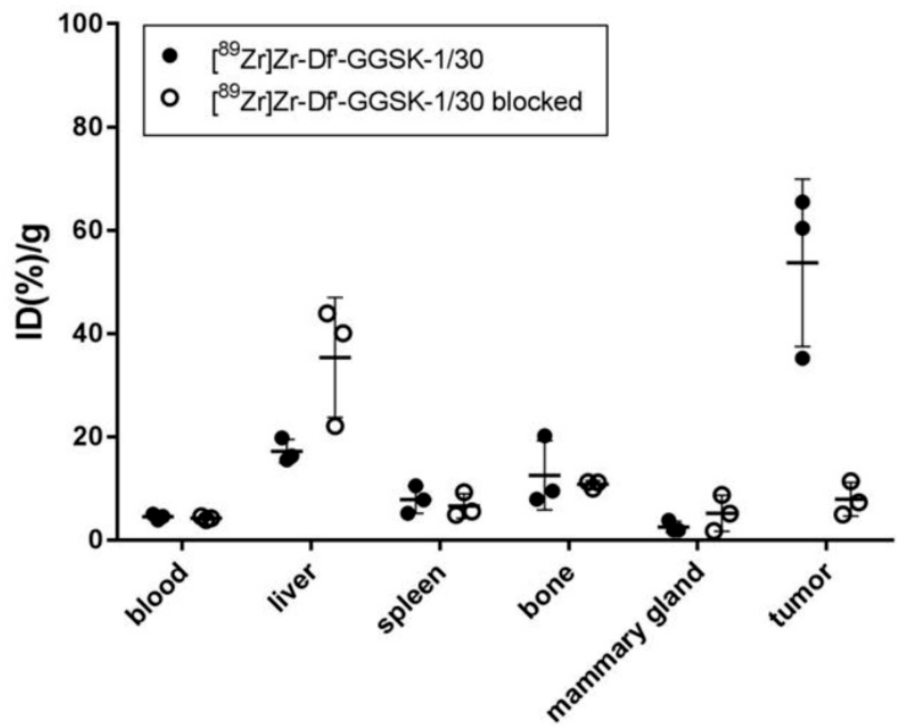

B

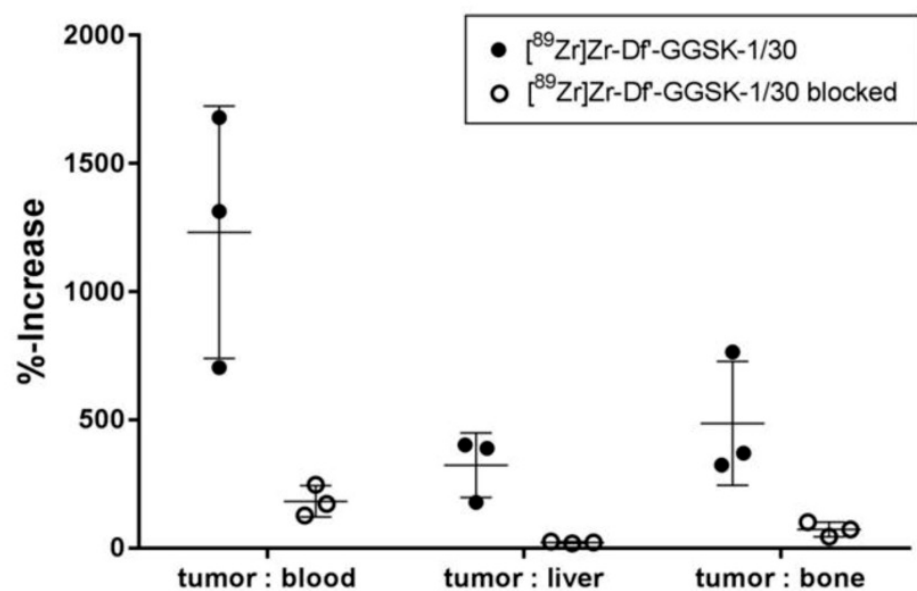

C

[ $\left.{ }^{89} \mathrm{Zr}\right] \mathrm{Zr}$-DFO-BZ-NCS-GGSK-1/30

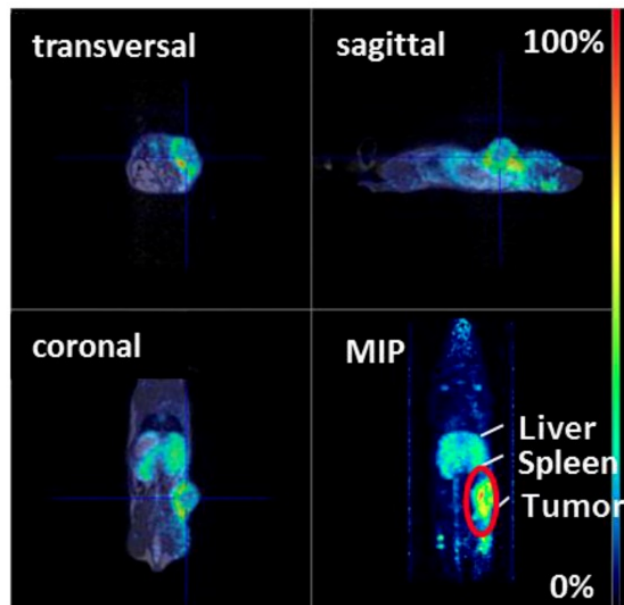

[ ${ }^{89} \mathrm{Zr}$ ]Zr-DFO-Bz-NCS-GGSK-1/30 blocked

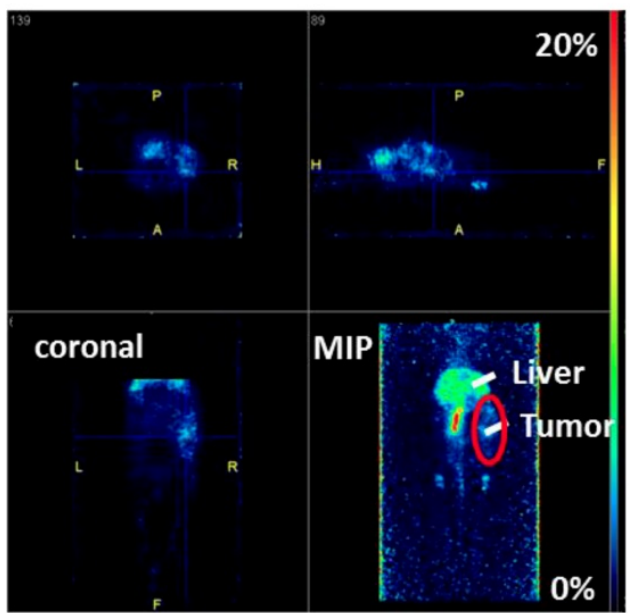

Figure 6: Selective binding of [ $\left.{ }^{89} \mathrm{Zr}\right] \mathrm{Zr}$-Df-GGSK-1/30 to hu(TA)MUCl expressed by PyMTxhuMUCl tumors. HuMUCl-transgenic mice bearing a PyMTxhuMUC1 breast tumor transplant subcutaneously on the right flank were treated i.p. with [ ${ }^{89} \mathrm{Zr}$ ]Zr-Df-GGSK-1/30 (80 $\mu \mathrm{g}, 2.5 \mathrm{MBq}$, black dots: $\bullet$ ), previously saturated with 1200 molar excess of the corresponding glycopeptide: [89Zr]Zr-Df-GGSK-1/30 blocked (50 $\mu \mathrm{g}, 0,46 \mathrm{MBq}$, open circles: $\bigcirc)$. After $72 \mathrm{~h}$ the distribution of the radioconjugate was determined (A), the tumor/non-target-tissue ratios $(B)$ were determined $(I D(\%) / g($ tumor $): \operatorname{ID}(\%) / g(b l o o d$, liver, bone)* $100=\%$-increase) and PET imaging was performed with representative mice (B). Maximum Intensity Projections (MIPs) are shown. Abbr.: tu: tumor, ki: kidney, li: liver. 


\section{Discussion}

Early and specific detection of breast cancer remains a challenge in oncology. Intensive efforts are being made to identify the biological processes and new targets for TNBC. Molecular imaging of these targets may aid target identification, drug development, and in predicting and evaluating response to therapy (20). GGSK-1/30 is characterized by the fact that it exclusively recognizes a clearly defined synthetic MUC1-derived glycopeptide which was concomitantly demonstrated to block its binding to human breast cancer cells (29). In comparison SM3 and HMFG1 were induced against partial deglycosylated MUC1 from human milk. The binding epitope is the PDTRP amino acid sequence of the MUC1 tandem repeat. Due to the microheterogeneity of these antigens, the induced antibodies are not sufficiently specific to differentiate between physiological MUC1 and (TA) MUC1 $(30,31)$. The exceptional specificity of GGSK-1/30 for $\mathrm{hu}(\mathrm{TA}) \mathrm{MUC1}$ combined with radiolabeling to the long lived isotope ${ }^{89} \mathrm{Zr}$ allowed the generation of an innovative diagnostic tool characterized by high tumor accumulation to visualize hu(TA)MUC1 expression on breast cancer manifestations via PET imaging technology. In addition, GGSK-1/30 exhibited much higher and more specific tumor enrichment levels than previously reported for other anti-MUC1 mAbs $(32,33)$. With these characteristics GGSK-1/30 represents a new promising tool concerning clinical studies for molecular imaging of breast cancer that might also be used for radiotherapeutic approaches since the $\mathrm{mAb}$ meets the key foundations for effective radioimmunotherapy: A high and tumor-specific accumulation of the radiopharmaceutical, as well as a low dose rate for the patients to avoid collateral damage from surrounding healthy tissue (34). Especially, recent studies have shown that (TA)MUC1 due to its strong expression in HR-positive (35,36), HER2/neu-positive breast tumors (14) and in TNBCs (11), is crucially involved in the development of resistance to the clinically used adjuvant therapies (tamoxifen, trastuzumab, systemic chemotherapy). The combinatory therapeutic use of anti-(TA)MUC1 antibody drugs with common therapeutic agents in adjuvant therapy could therefore increase their clinical effect.

\section{Conclusion}

$\mathrm{Hu}$ (TA)MUC1 is a tumor-specific antigen on breast cancer cells with an exceptionally high diagnostic and potential prognostic value/importance. Immunizations against hu(TA)MUC1 enabled us to generate a unique antibody that specifically recognizes hu(TA)MUC1 glycopeptides on breast cancer cells. The specific immunohistochemical staining of breast cancer tissue with the mAb GGSK-1/30 confirmed that (TA)MUC1 represents a promising marker for diagnosis and most likely prognosis (37). Especially, due to its overexpression in $90 \%$ of all breast cancer patients $(10,38)$ and in $94 \%$ of TNBC patients, (11) as well as the clear association of high expression with metastases and poor survival (39). The radiolabeled derivative [ $\left.{ }^{89} \mathrm{Zr}\right] \mathrm{Zr}-\mathrm{Df}^{\prime}$-GGSK-1/30 demonstrated high in vivo stability and highly selective and tumor-specific accumulation which resulted in high contrast PET imaging. Thus, GGSK-1/30 represents a promising PET-tracer for clinical studies on molecular imaging in early diagnosis and/or in therapyaccompanying control examinations of breast cancer patients undergoing systemic therapies. In conclusion, the mAb GGSK-1/30 represents a platform, which can be used (i) as a diagnostic tool for the detection of hu(TA)MUC1 in early breast cancer diagnosis, (ii) as a prognostic biomarker, (iii) as a companion diagnostic during therapy and (iv) in future perspective in radioimmunotherapy.

\section{Supplementary Material}

Supplementary figures and tables.

http://www.medsci.org/v16p1188s1.pdf

\section{Abbreviations}

MUC1: mucin1; hu: human; TA: tumorassociated; ID: injected dose; $\mathrm{mAb}$ : monoclonal antibody.

\section{Acknowledgment}

The authors thank Markus Glaffig for supplying the glycopeptide for blocking experiments. We thank Nicole Bausbacher for her kind help during PET/MRT studies.

\section{Financial Disclosure}

This project was financially supported by the SFB1066 and the "Inneruniversitäre Forschungsförderung" of the JGU Mainz.

\section{Disclaimer}

All authors consent to publication. The study has been approved by the institutional review board and all subjects signed an informed consent form. No other potential conflict of interest relevant to this article was reported.

\section{Competing Interests}

The authors have declared that no competing interest exists. 


\section{References}

1. Lafourcade A, His M, Baglietto L, Boutron-Ruault M-C, Dossus L, Rondeau V. Factors associated with breast cancer recurrences or mortality and dynamic prediction of death using history of cancer recurrences: the French E3N cohort. BMC Cancer [Internet]. 2018;18(1):171.

2. Braden A, Stankowski R, Engel J, Onitilo A. Breast Cancer Biomarkers: Risk Assessment, Diagnosis, Prognosis, Prediction of Treatment Efficacy and Toxicity, and Recurrence. Curr Pharm Des [Internet]. 2014;20(30):4879-98.

3. Alwan NAS, Tawfeeq FN, Muallah MH, Sattar SA, Saleh WA. The Stage of Breast Cancer at the Time of Diagnosis: Correlation with the Clinicopathological Findings among Iraqi Patients. J Neoplasm [Internet]. 2017;2(3:11):1-9.

4. Masood S. Breast cancer subtypes: morphologic and biologic characterization. Womens Health (Lond Engl) [Internet]. 2016;12(1):103-19.

5. Gaidzik N, Kaiser A, Kowalczyk D, Westerlind U, Gerlitzki B, Sinn HP, et al. Synthetic antitumor vaccines containing MUC1 glycopeptides with two immunodominant domains-induction of a strong immune response against breast tumor tissues. Angew Chem Int Ed Engl [Internet]. 2011;50(42):9977-81.

6. Nath S, Mukherjee P. MUC1: a multifaceted oncoprotein with a key role in cancer progression. Trends Mol Med [Internet]. 2014;20(6):332-42.

7. Kaiser A, Gaidzik N, Westerlind U, Kowalczyk D, Hobel A, Schmitt E, et al. A Synthetic Vaccine Consisting of a Tumor-Associated Sialyl-T N -MUC1 Tandem-Repeat Glycopeptide and Tetanus Toxoid: Induction of a Strong and Highly Selective Immune Response. Angew Chemie Int Ed [Internet]. 2009;48(41):7551-5.

8. Taylor-Papadimitriou J, Burchell J, Miles D. W, Dalziel M. MUC1 and cancer. Biochim Biophys Acta - Mol Basis Dis [Internet]. 1999;1455(2):301-13.

9. Hanisch FG. O-glycosylation of the mucin type. Biol Chem. 2001;382(2):143-9.

10. Miller-Kleinhenz JM, Bozeman EN, Yang L. Targeted nanoparticles for image-guided treatment of triple-negative breast cancer: clinical significance and technological advances. Wiley Interdiscip Rev Nanomedicine Nanobiotechnology [Internet]. 2015;7(6):797-816.

11. Siroy A, Abdul-Karim FW, Miedler J, Fong N, Fu P, Gilmore H, et al. MUC1 is expressed at high frequency in early-stage basal-like triple-negative breast cancer. Hum Pathol [Internet]. 2013;44(10):2159-66.

12. Palitzsch B, Gaidzik N, Stergiou N, Stahn S, Hartmann S, Gerlitzki B, et al. A Synthetic Glycopeptide Vaccine for the Induction of a Monoclonal Antibody that Differentiates between Normal and Tumor Mammary Cells and Enables the Diagnosis of Human Pancreatic Cancer. Angew Chemie - Int Ed [Internet]. 2016;55(8):2894-8

13. Brockhausen I, Yang J-MM, Burchell J, Whitehouse C, Taylor-Papadimitriou J. Mechanisms underlying aberrant glycosylation of MUC1 mucin in breast cancer cells. Eur J Biochem [Internet]. 1995;233(2):607-17.

14. Sinn B V., Von Minckwitz G, Denkert C, Eidtmann H, Darb-Esfahani S, Tesch $\mathrm{H}$, et al. Evaluation of Mucin-1 protein and mRNA expression as prognostic and predictive markers after neoadjuvant chemotherapy for breast cancer. Ann Oncol [Internet]. 2013;24(9):2316-24.

15. Guy CT, Cardiff RD, Muller WJ. Induction of mammary tumors by expression of polyomavirus middle $\mathrm{T}$ oncogene: a transgenic mouse model for metastatic disease. Mol Cell Biol [Internet]. 1992;12(3):954-61.

16. Perk LR, Vosjan MJWD, Visser GWM, Budde M, Jurek P, Kiefer GE, et al. P-Isothiocyanatobenzyl-desferrioxamine: A new bifunctional chelate for facile radiolabeling of monoclonal antibodies with zirconium-89 for immuno-PET imaging. Eur J Nucl Med Mol Imaging. 2010;37(2):250-9.

17. Vosjan MJWD, Perk LR, Visser GWM, Budde M, Jurek P, Kiefer GE, et al. Conjugation and radiolabeling of monoclonal antibodies with zirconium-89 for PET imaging using the bifunctional chelate p-isothiocyanatobenzyldesferrioxamine. Nat Protoc [Internet]. 2010;5(4):739-43.

18. Stergiou N, Gaidzik N, Heimes A-S, Dietzen S, Besenius P, Jäkel J, et al. Reduced breast tumor growth after immunization with a tumor-restricted MUC1 glycopeptide conjugated to tetanus toxoid. Cancer Immunol Res [Internet]. 2018; canimm.0256.2018.

19. Perou CM, Sørlie T, Eisen MB, van de Rijn M, Jeffrey SS, Rees CA, et al. Molecular portraits of human breast tumours. Nature [Internet]. 2000;406(6797):747-52.

20. Kurihara H, Shimizu C, Miyakita Y, Yoshida M, Hamada A, Kanayama Y, et al. Molecular imaging using PET for breast cancer. Breast Cancer [Internet]. 2016;23(1):24-32

21. Lei Lei, Xiaojia Wang ZC. PET/CT Imaging for Monitoring Recurrence and Evaluationg Response to Treatment in Breast Cancer. Adv Clin Exp med [Internet]. 2016;25(2):377-82.

22. Rowse GJ, Tempero RM, VanLith ML, Hollingsworth MA, Gendler SJ. Tolerance and immunity to MUC1 in a human MUC1 transgenic murine model. Cancer Res [Internet]. 1998;58(2):315-21.

23. Link JM, Krohn KA, Eary JF, Kishore R, Lewellen TK, Johnson MW, et al. Zr-89 for antibody labeling and positron emission tomography. J Label Compd Radiopharm. 1986;23(10):1297-8.

24. O'Brien HAJ. Overview of radionuclides useful for radioimmunoimaging/radioimmunotherapy and current status of preparing radiolabeling antibodies. Radioimmunoimaging Radioimmunother. 1983;17(4).

25. Bensch F, Smeenk MM, van Es SC, de Jong JR, Schröder CP, Oosting SF, et al. Comparative biodistribution analysis across four different $89 \mathrm{Zr}$-monoclonal antibody tracers-The first step towards an imaging warehouse. Theranostics [Internet]. 2018;8(16):4295-304.

26. Verel I, Visser GWM, Boellaard R, Walsum Stigter-Van M, Snow GB, Van Dongen GAMS. Zr-89 Immuno-PET: Comprehensive Procedures for the Production of Zr-89-Labeled Monoclonal Antibodies. J Nucl Med. 2003;44:1271-81.

27. Holland JP, Caldas-Lopes E, Divilov V, Longo VA, Taldone T, Zatorska D, et al. Measuring the Pharmacodynamic Effects of a Novel Hsp90 Inhibitor on HER2/neu Expression in Mice Using Zr-89-DFO-Trastuzumab. PLoS One. 2010;5(1):e8859.

28. Holland JP, Divilov V, Bander NH, Smith-Jones PM, Larson SM, Lewis JS. Zr-89-DFO-J591 for ImmunoPET of Prostate-Specific Membrane Antigen Expression In Vivo. J Nucl Med. 2010;51(8):1293-300.

29. Palitzsch B, Glaffig M, Kunz H. Mucin glycopeptide-protein conjugates Promising antitumor vaccine candidates. Isr J Chem. 2015;55(3-4):256-67.

30. Berry N, Jones DB, Smallwood J, Taylor I, Kirkham N, Taylor-Papadimitriou J. The prognostic value of the monoclonal antibodies HMFG1 and HMFG2 in breast cancer. Br J Cancer [Internet]. 1985;51(2):179-86.

31. Lalani E-N, Berdichevsky F, Boshell M, Shearer M, Wilson D, Stausss H, et al. THE JOURNAL OF BIOLOGICAL CHEMISTRY Expression of the Gene Coding for a Human Mucin in Mouse Mammary Tumor Cells Can Affect Their Tumorigenicity*. 1991;266(23):15420-6.

32. Alirezapour B, Rasaee MJ, Jalilian AR, Rajabifar S, Mohammadnejad J, Paknejad M, et al. Development of [64Cu]-DOTA-PR81 radioimmunoconjugate for MUC-1 positive PET imaging. Nucl Med Biol [Internet]. 2016;43(1):73-80.

33. Schuhmacher J, Klivényi G, Kaul S, Henze M, Matys R, Hauser H, et al. Pretargeting of human mammary carcinoma xenografts with bispecific anti-MUC1/anti-Ga chelate antibodies and immunoscintigraphy with PET. Nucl Med Biol [Internet]. 2001;28(7):821-8.

34. Rutqvist LE. Radiation therapies for breast cancer: current knowledge on advantages and disadvantages. Recent Results Cancer Res [Internet]. 1993;127:119-27

35. Zaretsky JZ, Barnea I, Aylon Y, Gorivodsky M, Wreschner DH, Keydar I. MUC1 gene overexpressed in breast cancer: structure and transcriptional activity of the MUC1 promoter and role of estrogen receptor alpha (ERa) in regulation of the MUC1 gene expression. Mol Cancer [Internet]. 2006;5(1):57.

36. McGuckin MA, Quin RJ, Ward BG. Progesterone stimulates production and secretion of MUC1 epithelial mucin in steroid-responsive breast cancer cell lines. Int J Oncol [Internet]. 1998;12(4):939-45.

37. Yang $\mathrm{E}, \mathrm{Hu} \mathrm{XF}$, Xing PX. Advances of MUC1 as a target for breast cancer immunotherapy. Histol Histopathol [Internet]. 2007;22(8):905-22.

38. Ho SB, Niehans GA, Lyftogt C, Yan PS, Cherwitz DL, Gum ET, et al. Heterogeneity of mucin gene expression in normal and neoplastic tissues. Cancer Res [Internet]. 1993;53(3):641-51.

39. McGuckin MA, Walsh MD, Hohn BG, Ward BG, Wright RG. Prognostic significance of MUC1 epithelial mucin expression in breast cancer. Hum Pathol [Internet]. 1995;26(4):432-9. 